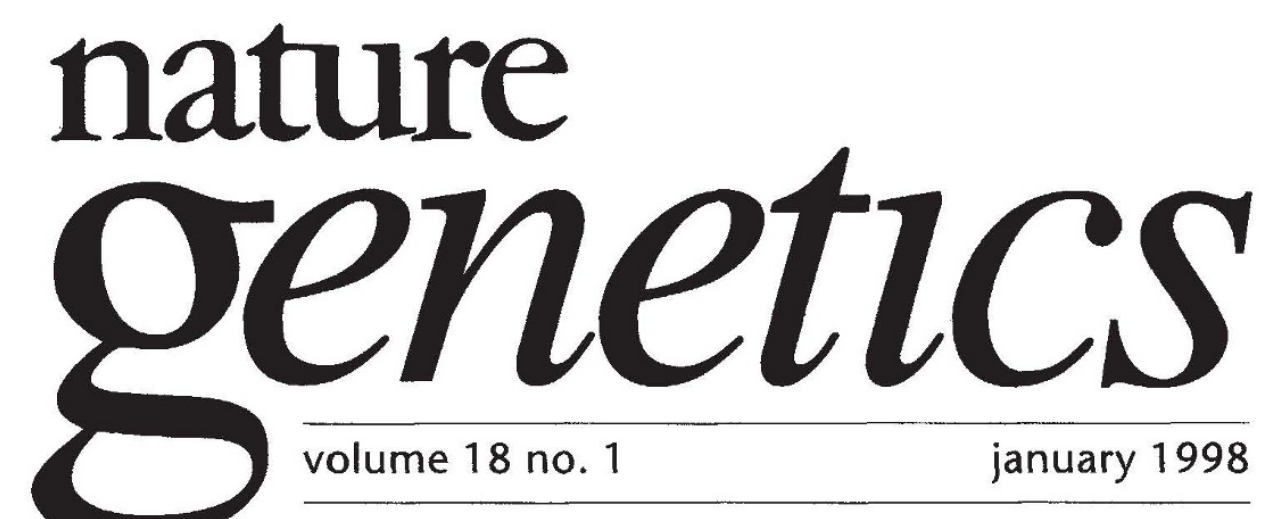

\title{
A taste of things to come
}

Tomorrow's menu may well include anti-cancer tomatoes, vitamin-supplemented fruit and fast-growing fish, thanks to genetic engineering and in response to an insatiable consumer appetite for tasty, cheap, healthy, low-calorie, long-lasting and environmentally friendly foods (for what more could one ask?). On the other hand, high-tech tampering of the edible raises, inevitably, public anxiety and concern. An exhibition at London's Science Museum entitled "Future Foods?", sponsored by the British Food and Drink Federation, the Biotechnology and Biological Sciences Research Council, and the Ministry of Agriculture, Fisheries and Food, aims to educate the public about the science behind genetic engineering of foods, crops and agricultural animals. Visitors learn about the step-by-step process of introducing an insect-resistance gene from the invincible snowdrop to the vulnerable cauliflower and can play a space-invaders-type game to genetically defend a virtual crop against an invasion of deadly caterpillars. But with the genegun pointed at food, there is the danger of shooting first and asking questions later. With this in mind, the exhibition raises the issues of consumer choice and explores the controversy surrounding the safety of genetically engineered food, possible effects on the environment and the ethics of manipulating genes.

Greenpeace, an outspoken opponent of genetic engineering, is prominently listed as a supporter of the exhibition and has contributed some of its campaign material. Having seen the entire exhibition, however, Greenpeace U.K. spokesperson Doug Parr complained that it "underplays environmentalist concerns and is pro-industry". Greenpeace is concerned that visitors could miss the warnings of potential risks and instead see genetic engineers as heroes who kill bugs and make food tastier. Professor Dick Flavell, director of the John Innes Centre for Genetic Research defends the show, emphasizing that "the purpose of the exhibition is not to provide the answers to everything, but to bring information and debate into the public domain".

The problem, as is so often the case, is a blurring of the issues. Neither the benefits nor the risks are well-defined. There are many examples whereby genetic modification of food offers potential future benefits, such as the development of 'edible vaccines'. A vaccine against diarrhoea - a life-threatening disease in developing countries-is currently under development at the Boyce Thompson Institute (BTI), Ithaca, New York. Charles Arntzen and co-workers have engineered potatoes expressing vaccines for bacterial and viral diarrhoea and, after successful immunization of mice fed with the transgenic spuds, they are now conducting an FDA-approved trial in human volunteers. But raw potatoes are not very palatable, and efforts are underway to produce the vaccines in bananas instead. Banana vaccine plants would eventually be grown locally in developing countries, and the fruit may be ready to peel in less than five years. "Our target is to 


\section{IMAGE \\ UNAVAILABLE FOR COPYRIGHT REASONS}

Santa Claus with test tube and labelling demand. Greenpeace/Novis try to get the price for a single-dose vaccine below ten cents, and I see absolutely no reason why we can't reach that goal and even better", claims Arntzen, who notes that "Putting vaccines into a food supply is a means of making it possible to get them to poor countries". Even skeptics will find it difficult to argue against a smart and cheap way to save childrens' lives in developing countries. But at the other end of the spectrum are alarming reports about the spread of resistance disease genes from genetically engineered crop species to their wild relatives, fueling environmentalists' concerns that entire ecosystems could be set off balance as a result of the release of genetically modified organisms.

One such organism is Monsanto's genetically engineered soybean, which is still making headlines in Europe. This product is not cheaper, healthier or better-tasting than normal soybeans, but rather is resistant to a particular herbicide (whose patent is held by Monsanto). The issues are complex. Monsanto claims that the modified soybeans will eventually be cheaper to produce and that the herbicide is environmentally friendly. But Monsanto is mixing these soybeans with unmodified ones, thereby eliminating the buyer's choice to purchase unaltered soya. Given Monsanto's expanding market share-it recently signed a deal with Millenium that gives it exclusive access to Millenium's genomics expertise for all crop plants in all countries-and that over $50 \%$ of processed foods contain soybean-derived ingredients, genetically engineered soya may be found in anything from chocolate bars to baby food. As a result, it will become increasingly difficult to distinguish between products derived from genetically-modified as opposed to unaltered plants.

Labelling — which would enable consumer choice — is hotly debated; should all foods that might contain products of genetic engineering be marked, or should there be labels for food produced without genetic manipulation? Greenpeace demands the segregation of genetically engineered and normal crops and a proper labelling system to trace genetically modified products from the source. The organization recently sent chocolate Santa Clauses to all members of the European Commision as well as the EU health and consumer affairs ministers. The Santas were labelled "We do not want to be surprised. We want to know! Greenpeace". Those recipients who were brave enough to eat the potentially genetically-engineered chocolate, found a test tube inside, symbolizing the "genetic experiment with our food". Earlier this year, the European Commission decided that all 'novel' foods need to be labelled, but it remains unclear what precisely constitutes a 'novel' food and how the labels should be worded. More recently, the Commission proposed that products derived from genetically engineered organisms must be labelled if they contain ingredients in which the modified DNA — or proteins altered as a consequence of the manipulation-can be detected. These proposals have generated more controversy than consensus. The food industry is confused about the nature of the testing requirements while environmentalist and consumer organisations argue that, under the proposed regulations, many products derived from genetically modified soybeans and maize ( $80 \%$, according to Greenpeace) would go unlabelled.

With some genetically modified foods already on the supermarket shelves, and with imminent mandatory labelling of some kind, European consumers will soon be able to vote with their shopping trolleys. However, to make an informed risk-benefit assessment of the product in hand, whether it be banana vaccines or herbicide-resistant soybeans, one must have digestible information on the science behind the technology and its potential dangers. Concerns about food safety have been an inherent part of our culture for millennia — consider the ancient religious bans on pork and shell-fish — and it is therefore vital that the public be informed and assured of the safety measures in place to oversee the introduction of genetically modified organisms into the environment as well as our food chain. Environmentalists, politicians, the food industry, consumer groups and the scientific community together face the challenge to educate and inform. An analysis of the risks and benefits must be provided for each individual example of genetically engineered food - and the balance may tip different ways for different applications of the same science and technology. 